\title{
Mechanism and Work of Kureha's Auto-Doffer
}

\author{
By Kazuhiko Takemura, Member, TMS
}

Kureha Spinning Co., Ltd., Osaka

\begin{abstract}
To trace the development of a full-automatic auto-doffer simple in mechanism and usuable in conventional spinning mills.
\end{abstract}

\section{The Doffer}

(1) The mechanism used is of an intermittent-movement and multi-spindle doffing system.

(2) The width of the doffer is $189 \mathrm{~mm}$.

(3) Conventional ring frames and buildings may be used if there is a space of more than $300 \mathrm{~mm}$ between the gear-end side (or out-end side) of a ring frame and a pillar.

(4) One person can move the auto-doffer if an auto-doffer carrier is used.

(5) The rails for the auto-doffer carrier need not be laid on the floor.

(6) The doffing time per cycle is about $8 \mathrm{sec} / 10 \mathrm{sps}$.

(7) The end-breakage rate for 10 ring frames as of June 1965 is about $2.5 \%$.

\section{Introduction}

Auto-doffers for spinning are now made in Japan and elsewhere. Thcy are very much in the limelight.

Labor needed for the fine spinning process accounts for about $40 \%$ of the total labor needed for a cotton spinning mill. Labor needed for the doffing process is about $30 \%$ of the labor for fine spinning process and is, therefore, about $12 \%$ of the total mill labor force.

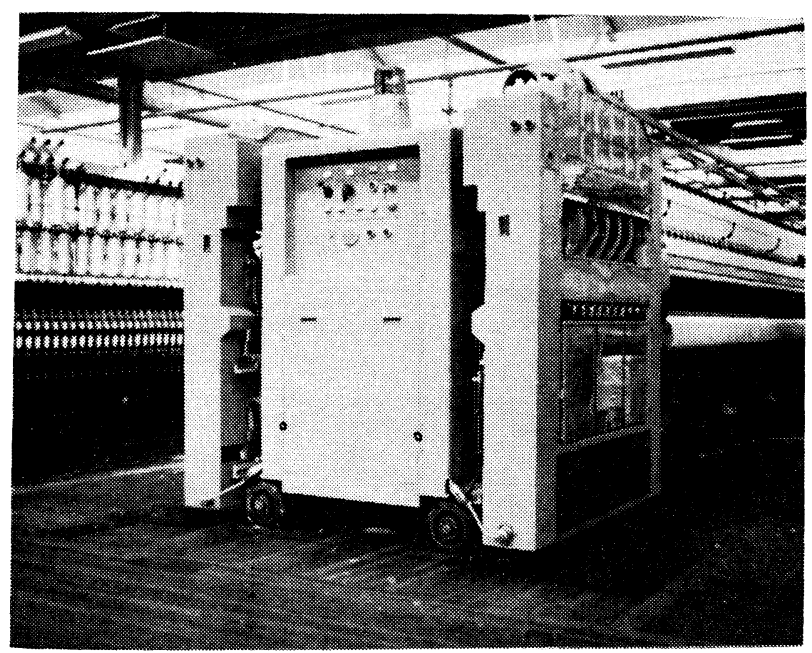

Fig. 1 A overall view of KAD
Use of an auto-doffer saves about $10 \%$ of labor per bale. The figure is too important to be overlooked, although the exact figure varies from mill to mill. The need for a labor-saving machine like this is unquestionably great, in view of the increasingly serious labor shortage facing the textile industry.

In 1958 we began developing an auto-doffer with the cooperation of Toyama Machine Co. for use as part of Kureha's Modernized Spinning System(KMS). We explained the idea of Kureha's Auto-Doffer (K.A.D.) in Vol. 17, No. 3, of the Journal of The Textile Machinery Society of Japan (Japanese edition). The present article describes the mechanism and work of K.A.D.

\section{Principle of Designing}

Kurena's Auto-Doffer is designed to be fit for use without modification of existing ring frames. The mechanism used is of an intermittent-movement and multi-spindle doffing system. The whole weight of the Auto-Doffer is supported by the upper and lower rails laid on the ring frame. The auto-doffer needs no rail on the floor and is not affected by the unevenness of the floor. An auto-doffer carrier is used to facilliate the entery of the A.D. into the ring frame. Successful efforts have been made to 
limit the width of A.D. to a minimum of $189 \mathrm{~mm}$ (see Fig. 2).

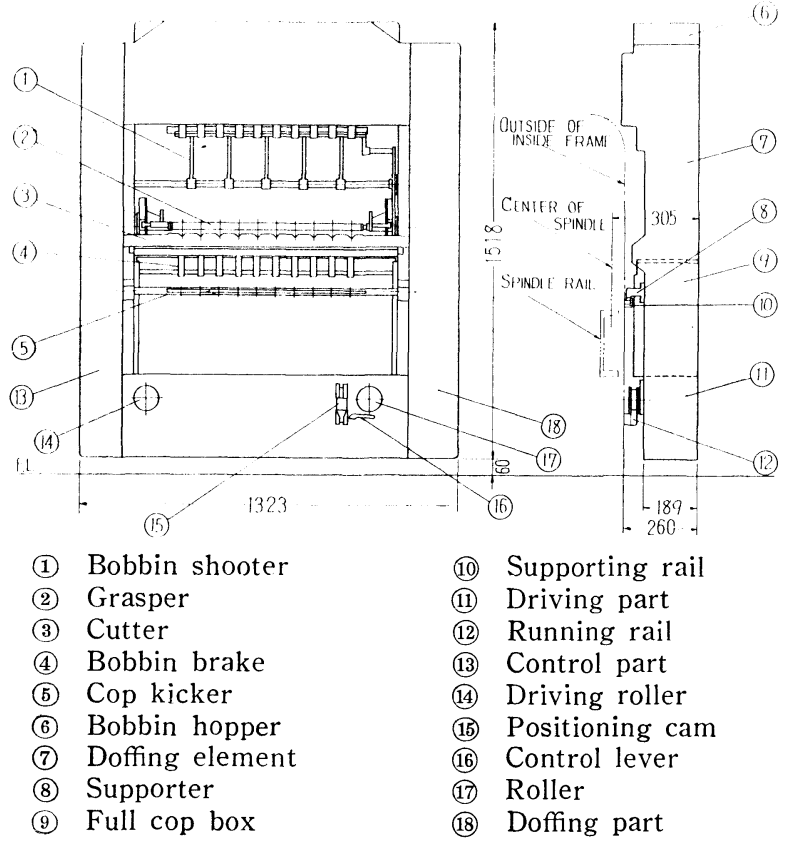

Fig- 2 Arrangement of KAD mechanism

\section{Mechanism and Action}

\section{3-1. Main Action}

(a) Two rails are laid on the spindle rail of the ring frame. The upper rail supports K.A.D., which runs on the lower rail. When K.A.D. comes above the doffing position guide, it touches a control lever, stops running and stays in the doffing position. (There is a control lever for each 10 spindles.)

Then K.A.D. begins doffing. Before that, the ring rail has to be raised $35-40 \mathrm{~mm}$ from the position in which bobbin-winding begins; and lappets should be slightly inclined.

Actions preparatory to grasping K.A.D. is as follows :

(i) A lappet-turning mechanism pushes up the lappets to facilitate ejection and insertion of bobbins.

(ii) A cop kicker revolves $90^{\circ}$ out of K.A.D., lifts the cops $15 \mathrm{~mm}$ and separates them completely from the spindles.

(iii) When these two actions are completed, a grasper lined with elastic rubber drops to the top of the cop and grasps the cop. The gripping power is supplied by oil-pressure and a spring. (see Fig. 3.)
The action of the grasper consists of three motions :

(i) The upward and downward movements of the grasper.

(ii) The entry and exit of the rack.

(iii) The grasper is pulled upward by the lever, which is actuated by a cam.

The cutter cuts yarn which is between cop and the spindle. Then the grasper releases the cop from its grasp.

\section{(b) Bobbin-shooting}

The mechanism of bobbin-shooting consists of a bobbin hopper and a bobbin shooter. The hopper is fixed on K.A.D. Ten or 11 bobbins are lined up in a column; $200-220$ bobbins in all, making up 20 columns, are placed in the hopper. Bobbins are supplied to the bobbin shooter by using two rows of hoppers per spindle, each bobbin being supplied with one rotation of the supply roller. These actions are made by oil pressure.

The bobbin shooter receives bobbins, which are supplied horizontally from the bobbin hopper, into the bobbin catcher and guides them on spindles, then free them and returns to its original position. (The shooter receives bobbins for 10 spindles at a time.) The bobbin shooter is fixed to the end of the arm which fixed on the rod, so that the bobbin catcher turns freely.
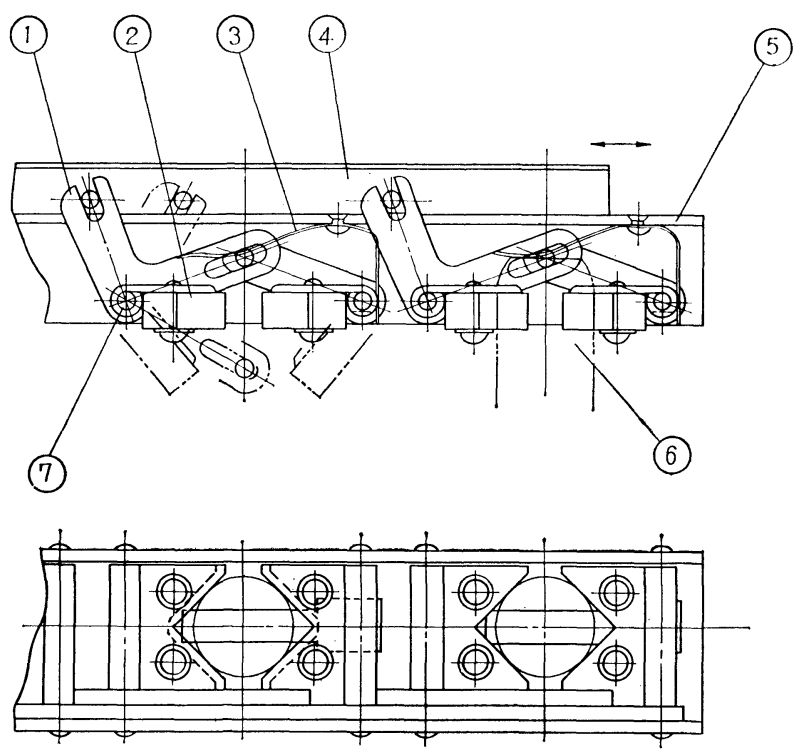
(1) Lever
(2) Elastic gum
(3) Spring
(5) Grasper
(6) Cop
(7) Fulcrum
(4) Rod

Fig. 3 Construction of the grasper 
When the bobbin shooter device receives bobbins from the hopper, the catcher is kept horizontal by the cam. Bobbins drop to the catcher and are pressed to the catcher by a spring-powered bobbin presser. The bobbin shooter holds and pushes out the bobbin by the force of the rotations of the rod of the catcher's arm. The bobbin then runs over the guide cam.

If bobbins drop too fast, the end-breakage rate is high. A bobbin brake is used to slow down the dropping of bobbins. When bobbins enter spindles, the bobbin presser arranges the tops of the bobbins in order (see Fig. 4).

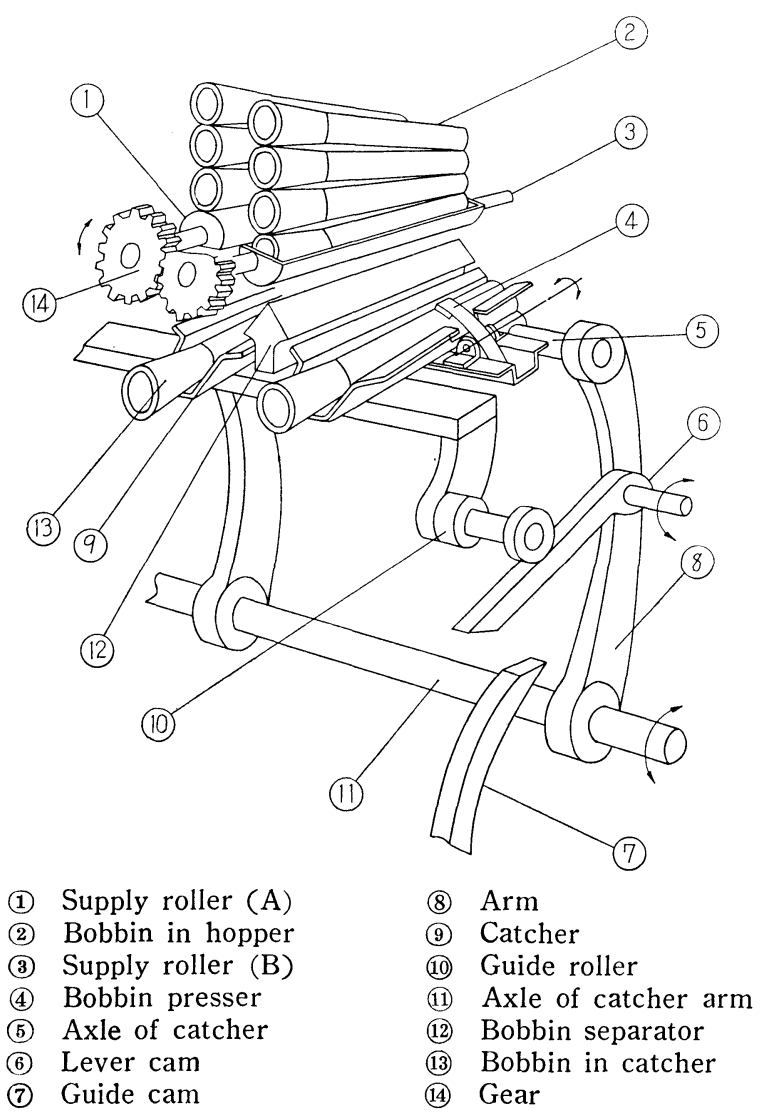

Fig. 4 Mechanism of cop supplying

\section{3-2. Auxiliary Actions}

\section{(a) K.A.D. carrier}

The carrier holds both sides of K.A.D. and runs on the gear-end side or out-end side at a right angle to the ring frame.

A guide rail, which is the same in width as the frame, is laid on the frame to hold the carrier and the frame in place. The carrier runs along the guide rail. There is no running rail for the carrier.
The running speed of the carrier is $25 \mathrm{~m} / \mathrm{min}$.

The operator of K.A.D. stops the carrier in the front of a ring frame needing doffing. $\mathrm{He}$ then operates a lever to:

(i) Hold the carrier and the ring frame in place.

(ii) Connect the rails for the carrier to the K.A.D. rails laid on the ring frame.

The upper and lower rails for the carrier move independently and can, therefore, be combined per. fectly, no matter what the floor condition is.

A carrier which automatically stops in front of a ring frame needing doffing can be built if required.

The K.A.D. carrier is operated by $\mathrm{AC} 220 \mathrm{~V}$. The operator couples the spinning frame rail with the carrier rail. Then he inserts K.A.D. into the ring frame by pushing a button.

When K.A.D. has completed its work, it returns to the carrier, automatically cutting off its power source. The carrier is removed from the ring frame and its upper and lower rails are separated. These operations are made by pushing buttons. (see Fig. 5.)

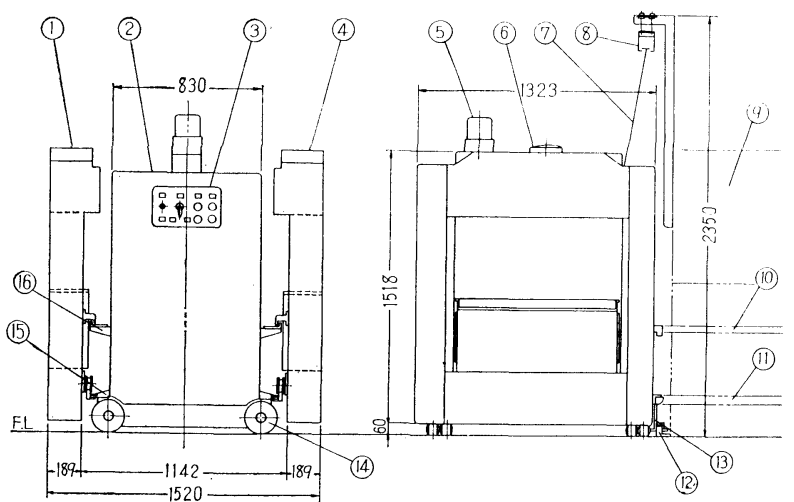

$\begin{array}{ll}\text { (1) } & \text { L. H AD } \\ \text { (2) KAD carrier } \\ \text { (3) Carrier panel } \\ \text { (4) R.H AD } \\ \text { (5) Lamp } \\ \text { (6) Horn } \\ \text { (7) Cord } \\ \text { (8) Trolley }\end{array}$

Fig. 5 Sketch of KAD
The way K.A.D. is built makes it difficult to make a large space for the packaging of bobbins. As an alternative, they are packaged in 10 layers, 20-21 bobbins per layer. To make bobbins drop in a uniform height, they are dropped in a fixed quantity with each descent of a bobbin-pressing plate. 


\section{Operation}

4-1. Preparations for Doffing

(a) Prepare K.A.D. for the following actions:

(i) Put bobbins in the hopper.

(ii) Replace the cop box.

(iii) Shift the carrier to the front of the ring frame needing doffing.

(iv) Place the carrier on the ring frame.

(b) Prepare the ring frame for doffing by: ends.

(i) Lowering the ring rail and building up cops

(ii) Raising the ring rail and turning the lappets.

\section{4-2. Doffing}

(a) Doff the ring frame by:

(i) Bringing the lappets back to their original position and adjusting the rail accordingly.

(ii) Switching back the hank-meter to zero.

This completes doffing of one ring frame. Then : frame.

(ii) Remove the carrier from the doffed ring

(iv) Shift the carrier to the next ring frame needing doffing.

(Other actions are automatic.)

\section{Capacity and Doffing Time}

The present average speed of doffing by K.A.D. is $13 \mathrm{cops} / \mathrm{sec}$ ( 1 cycle is $8 \mathrm{sec} / 10 \mathrm{sps}$.). The running speed of the auto doffer is $18 \mathrm{~m} / \mathrm{min}$., it is the same in doffing and in returning to the carrier. Plans are afoot to make a new K.A.D. capable of $6 \mathrm{sec} / 10 \mathrm{sps}$. per cycle and a running speed of 25 $\mathrm{m} / \mathrm{min}$. The running speed of the K.A.D. carrier is $25 \mathrm{~m} / \mathrm{min}$. The time required to combine the carrier's rails and the frame rail and to fix the position of the carrier is $25 \mathrm{sec}$. on the average. The time required for 1 cycle of K.A.D. (including getting the ring frame ready, operation of K.A.D., bobbin supply and action of the cop box) is, at present, about $6.5 \mathrm{~min}$.

The capacity of K.A.D. varies with operating conditions, but doffing 50 ring frames is possible.
The average rate of end-breakage rate for about 300 doffings of 40 s yarn is :

End-breakage during doffing $1.5 \%$

End-breakage during running $1.0 \%$

Total

$2.5 \%$

The rate of end-breakage during running varies with the condition of the frame and its operating rate.

\section{Other Information}

(a) The modifications and attachments for the ring frame.

(i) The ring frame need not be modified unless it has a protrusion which is wider than the width of inside frame.

(ii) At least these three attachments are needed for the ring frame:

1) A running rail and a supporting rail for K.A.D. have to be laid on the spindle rail of the frame.

2) A K.A.D. carrier guide has to be laid on the gear-end side (or out end side) of the floor.

3) A power-snpply cord for the K.A.D. carrier.

(iii) Other useful attachments

1) A hank-meter, an automatic device to lower the ring rail and a frame-stopping device.

2) Automatic devices (a) to raise the ring rail, (b) to turn the lappets and (c) for 0 back of the hank-meter.

3) An automatic starting device and a magnetic indicator for winding up of K.A.D.'s powersupply cord.

(b) History of development of K.A.D. and patents

We begin to develop K.A.D. jointly with Toyama machine Co. in December 1962 . K.A.D. was modified on several occasions between April 1964 and April 1965 in the Inami mill of our company. Since May 1965, 13 K.A.D.'s have been at work in our Shokawa mill.

Ten Japanese patents have been obtained for K.A.D. :

(6 ordinary patents and 4 "utility" patents)

Thirty-four new patents have been arplied for: (15 ordinary patents and 19 "utility" patents) 